\title{
Analysis of the Earth's magnetosphere states using the algorithm of adaptive construction of hierarchical neural network classifiers
}

\author{
Sergey Dolenko*, Vsevolod Svetlov, Igor Isaev, and Irina Myagkova \\ D.V.Skobeltsyn Institute of Nuclear Physics, M.V.Lomonosov Moscow State University, \\ 119191 Moscow, Russia
}

\begin{abstract}
This paper presents analysis of the results of clusterization of the array of increases in the flux of relativistic electrons in the outer radiation belt of the Earth by two clustering algorithms. One of them is the algorithm for adaptive construction of hierarchical neural network classifiers developed by the authors, applied in clustering mode; the other one is the well-known k-means clusterization algorithm. The obtained clusters are analysed from the point of view of their possible matching to characteristic types of events, the partitions obtained by both methods are compared with each other.
\end{abstract}

\section{Introduction}

The dynamics of the system "interplanetary space -solar wind -magnetosphere of the Earth" can be described by a multivariate time series, which includes the parameters of the interplanetary magnetic field (IMF) (IMF vector modulus and its three components); velocity, density and temperature of the plasma of the solar wind (SW); geomagnetic indices: Dst, Kp and AE. The outer Earth's radiation belt (OERB) is a part of the inner magnetosphere, and it is also a complex dynamic system, whose parameters (e.g., the intensity of the particle flux and the position of the boundaries) can greatly vary depending on the variations of IMF and SW components (in particular the speed of SV), as well as on geomagnetic perturbation.

The level of perturbation of the Earth's magnetosphere is estimated by using geomagnetic indices - Dst, Kp, AE-AL. The amplitude of planetary perturbations during geomagnetic storms, embracing the entire magnetosphere, is characterized by the amplitude of Dst-variation, which represents the maximum deviation of the variation of Earth's magnetic field from the quiet level, averaged over the values measured at the control chain of magnetic stations located at lower latitudes, i.e. near the equator [1]. Information about the experimentally measured values of Dst variation is provided by the branch of the World Data Center located in Kyoto (Japan) (http://wdc.kugi.kyoto-u.ac.jp/dstdir/index.html [2]). Geomagnetic disturbances can be caused as by reaching the Earth's orbit coronal mass ejections (CMEs), associated with solar flares, as by high-speed SW streams emerging from

\footnotetext{
Corresponding author: dolenko@srd.sinp.msu.ru
} 
coronal holes. A necessary condition for the development of a magnetic storm is the presence of Southern (negative) IMF component, which enables energy transfer from the solar wind to the magnetosphere [3].

Disturbances of Earth's magnetic field (geomagnetic perturbations), called magnetic storms, are one of the major manifestations of space weather. At present, as space weather one usually understands the processes and phenomena occurring in the Sun, in the solar wind, the Earth's magnetosphere and ionosphere, which can affect the operation of onboard and ground-based technological systems, as well as the health and well-being of people. Experimental studies show that geomagnetic disturbances have a direct influence on the state of the near-earth space (NES) [4], e.g., its radiation state, because the flux of relativistic electrons (RE) in the OERB typically increases by an order of magnitude and more (see [5-6] and the references therein). Extremely high RE flux can cause failures in electronic circuits of the apparata located onboard the spacecraft [7]. In addition, strong magnetic storms can cause disruption of telegraph lines and radio communications, pipelines, power lines and power grids [1].

From the foregoing it follows that the detailed study of processes occurring in the magnetosphere of the Earth, is a very important task - both from a fundamental point of view with the aim of understanding the processes of particle acceleration in the NES, and to improve methods of forecasting the values of its parameters, including those using selection of its different states. The latter can be accomplished using data clusterization algorithms.

\section{Algorithm of adaptive construction of hierarchical neural network classifiers}

In their laboratory, the authors of this study developed the algorithm of adaptive construction of hierarchical neural network classifiers (HNNC), which is a method of construction of a neural network tree, allowing one to reduce the solution of a complex problem of classification to a sequential or parallel solution of several problems of smaller complexity. The approach consists in an iterative analysis of the responses of a neural network and in adjusting its desired answers in the process of training. As the result of such adjustment, "similar" classes are combined into one, in which the same output neuron corresponds to patterns of both merged classes. Thus, at each level of the hierarchy similar classes form clusters (groups), and the task of the model is to determine the number of the corresponding cluster. In fact, at the same time the algorithm solves the problem of clustering of the plurality of the desired original classes and the problem of classification of patterns as belonging to the created clusters. Thus by reducing the number of clusters and by strong simplification of the desired separating surface the requirements on the complexity of the used model are weakened, which in turn leads to the fact that this approach can be applied even on small sample sizes.

Recursive application of the algorithm allows building adaptively a hierarchical system of classification; the number of clusters at each level of the hierarchy, i.e. the number of classes recognized by each neural network, remains small. The algorithm for constructing the hierarchical neural network classifier, first presented in [8-9], was studied and described in [10-13]. This algorithm can be used as in the classification mode, as in clustering mode [14]; the latter variant was applied in the present study. 


\section{Data sources and preparation}

In this study, the SW and IMF data from the ACE (Advanced Composition Explorer) spacecraft located at the Lagrange L1 point, obtained by the SWEPAM and MAG instruments respectively, were used [15]. Processes in the chain "IMF - SW magnetosphere of the Earth" are described using multivariate time series, which includes the parameters of IMF - module of the IMF vector and its three components; the parameters of the SW - velocity, density and temperature of SW plasma, and the geomagnetic indices Dst and Kp obtained from the page providing data for geomagnetism (Geomagnetic Data Service) [16], and the RE flux of the outer ERB (flux of electrons measured in the experiments at the spacecraft of the GOES series [17]). The data range used was from October 22, 1997 (the beginning of data receipt from ACE) until March 2016.

Analysis of the states of the outer radiation belt of the Earth was performed on the basis of data on the flux of RE in the geostationary orbit. Each of the clustered patterns represented a description of an event of increasing of the flux of RE of the OERB with energy $>2 \mathrm{MeV}$. In the computational experiment, the results of which are presented below, each event is described by the values of the following attributes:

count - duration of the event in hours (minimum considered event duration 12 hours);

$\mathrm{e} 0$ - the average value of the RE flux over the last 3 hours prior to the event;

el_max - the maximum value of the RE hourly average flux during the time of the event;

el_min - the minimum value of the RE hourly average flux during the time of the event;

el_sum_average - average RE flux during the time of the event (integral RE flux over the time of the event, normalized to the duration of the event);

spd_max - maximum hourly SW velocity during the event;

Bz_maxabs - maximum modulo hourly average value of the z-component of the IMF;

B_magn_max - the maximum hourly average value of the IMF modulus during the event;

Kp_mean - the mean value of the geomagnetic index Kp during the event;

Dst_max - maximum modulo hourly value of the geomagnetic index Dst during the event;

AE_mean - the mean value of the geomagnetic index AE during the event.

Clustering of events was performed by the developed algorithm with the following parameters: 5 neurons in the hidden layer, learning rate 0.01 , time-out before the first class merging -1 epoch, time-out between class mergings -1 epoch, the threshold of neuron "voting" - 0.02. Clusterization into 5 clusters was obtained. As an alternative, we considered the division into the same number of clusters obtained by using the k-means algorithm.

\section{Results and discussion}

Due to the fact that the distribution for most of the considered input features has a shape far from Gaussian, the values of their mean and standard deviation are insufficient for a meaningful analysis of the clustering results. Fig. 1 (a, b) shows the histograms for several of the most informative of these input characteristics for the entire dataset and for each cluster individually. The left column shows the clustering obtained using the developed HNNC algorithm, the right column is the clustering obtained using the k-means algorithm. 


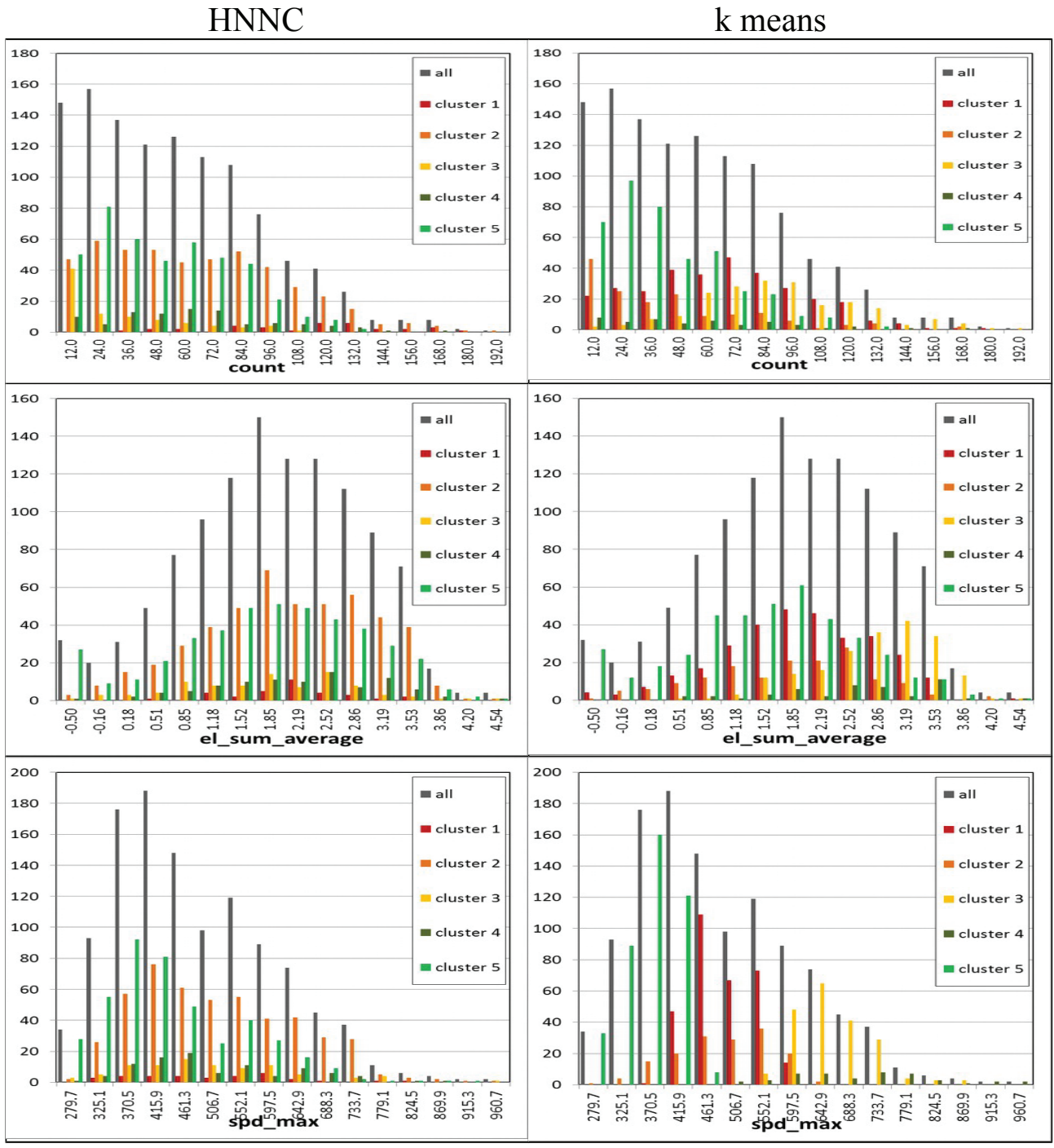

Fig. 1a. Histograms of distribution of several input features, for the entire dataset and for each cluster: 1) count - duration of the event in hours; 2) el_sum_average - average RE flux during the time of the event; 3) spd_max - maximum hourly SW velocity during the event. Left - clusterization, produced by the developed HNNC algorithm, right - clusterization, produced by the k-means algorithm. 


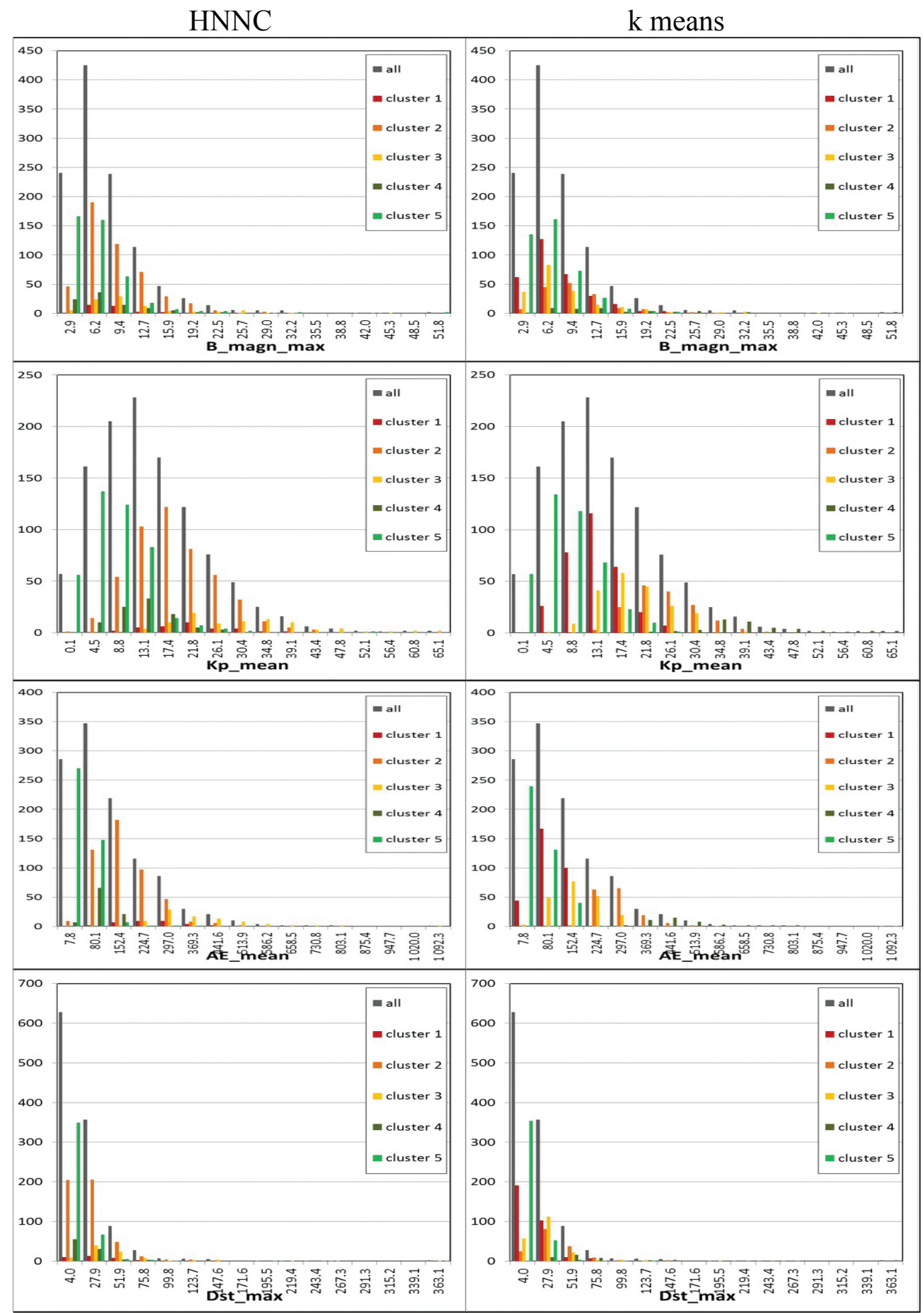

Fig. 1b. Histograms of distribution of several input features, for the entire dataset and for each cluster: 1) B_magn_max - the maximum hourly average value of the IMF modulus during the event; 2) Kp_mean - the mean value of the geomagnetic index Kp during the event; 3) AE_mean - the mean value of the geomagnetic index AE during the event; 4) Dst_max - maximum modulo hourly value of the geomagnetic index Dst during the event. 
From the above histograms it can be seen that both algorithms identified in cluster five (the green bars) short increases of the electron flux of medium intensity, occurring at a relatively low SW velocity and at small but non-zero amplitudes of geomagnetic indices. It can be assumed that the cause of such disturbances of the magnetosphere and of variations of the electron flux in the OERB caused by them may be slow coronal mass ejections coming to the Earth's orbit.

Further, from the analysis of the histograms obtained by the k-means method, it is clear that the events most protracted and having the most intense electron flux were selected by this method to the cluster number 3 (the yellow bars). They correspond to the maximum $\mathrm{SW}$ velocity and to relatively large values of the Kp index and of the amplitude of the IMF vector B. By comparison of the histograms we can assume that this cluster included the increases of the electron flux caused by powerful coronal mass ejections associated with solar flares.

The next one by the intensity of the events (and also by the SW velocity) is cluster 2 (depicted by orange bars). It is seen that in this cluster there are significantly less events than in clusters 3 and 1 . The histogram of the distribution of AE-index shows that this cluster includes events with maximum AE index, allowing one to suggest that for the events of cluster 2 the responsible processes are sub-storm processes which are characterized by large amplitude of the AE index.

Cluster 1 (dark red bars), larger by the number of events than clusters 2 and 3, includes events of medium intensity, emerging at SV with velocity less than for clusters 2 and 3. Most importantly, they occurred when the amplitude of the Dst index during the events of this cluster was low, in contrast to the amplitude of the $\mathrm{Kp}$ index, i.e. geomagnetic disturbances, characteristic for this cluster, can be characterized as high-latitude. Presumably this could be the disturbances caused by the arrival of high speed streams of solar wind from coronal holes.

Cluster 4 (grey-green) is too small, while the distribution of events in almost all parameters follows the distribution of cluster 2 . The reason why the algorithm highlighted some of these events into a separate cluster may be the subject of further research.

As for the results shown by the developed algorithm, from the analysis of the histograms it follows that the clusters $1,2,3$ according to the k-means algorithm were merged into a single cluster numbered 2; formally, the clustering result with HNNC was more "rough". On the other hand, the analysis of histograms shows that with the HNNC method, the events are well separated by the intensity of the Kp and AE indices, that is, the events caused by high-latitude perturbations were highlighted. A more detailed study of the obtained results and improving the performance of HNNC should also be continued.

\section{Conclusions}

Thus, in this study the array of the increases of the flux of relativistic electrons of the outer radiation belt of the Earth was split into 5 clusters characterizing the states of the Earth's magnetosphere different from the physical point of view, by the developed HNNC algorithm and by the k-means algorithm. The obtained clusters were analyzed from the point of view of their possible matching to distinctive types of events, and the two obtained clustering results were compared with each other.

This study was supported by the Russian Foundation for Basic Research (RFBR), grant No. 15-07-08975-a. 


\section{References}

1. L.L. Lasutin, World and polar magnetic storms (MSU, 2012)

2. Geomagnetic Equatorial Dst Index Home Page. URL: http://wdc.kugi.kyoto-u.ac.jp/dstdir/index.html

3. B.T. Tsurutani, W.D. Gonzalez, F. Tang et.al., J. Geophys. Res. A8, 8519 (1988)

4. I.N. Myagkova, Yu.S. Shugay, I.S. Veselovsky, and O.S. Yakovchouk, Solar Syst. Res., 47(2), 141 (2013).

5. R.H. Friedel, W.G.P. Reeves, T. Obara, J. Atmos. Solar. Terr. Phys. 64, 265 (2002).

6. R. Kataoka, Y. Miyoshi, Ann. Geophys. 25, 1335 (2008)

7. N. Iucci, A.E. Levitin, A.V. Belov et al., Space Weather, 3, S01001 (2005)

8. S.A. Dolenko, Yu.V. Orlov, I.G. Persiantsev, J.S. Shugai, E.K. Eremin, The 4th Open Russian-German Workshop on Pattern Recognition and Image Analysis, Valdai, Russia, March 4-8, pp. 45-49 (1996).

9. S.A. Dolenko, Yu.V. Orlov, I.G. Persiantsev, Ju.S. Shugai, and E.K. Eremin, Patt. Recogn. Image Anal. 7(1), 24 (1997).

10. S.A. Dolenko, Yu.V. Orlov, I.G. Persiantsev, Ju.S. Shugai, E.K. Eremin, Proceedings Fifth Int. Conf. on Artificial Neural Networks, Churchill College, University of Cambridge, UK, July 7-9, 1997. IEE Conference Publication, 440, 285 (1997).

11. S.A. Dolenko, Yu.V. Orlov, I.G. Persiantsev, Yu.S. Shugai, Patt. Recogn. Image Anal. 8(2), 122 (1998).

12. V.A. Svetlov, I.G. Persiantsev, J.S. Shugay, S.A. Dolenko, Opt. Mem. Neural Netw. (Inform. Opt.), 24(4), 288 (2015). DOI: 10.3103/S1060992X15040062

13. V.A. Svetlov and S.A. Dolenko, Opt. Mem. Neural Netw. (Inform. Opt.), 26(1), 40 (2017). DOI 10.3103/S1060992X17010076

14. V.A. Svetlov and S.A. Dolenko, XIVth All-Russian scientific conference "Neurocomputers and their application". Theses, p.55 (M., MGPPU, 2016).

15. World Data Center in Kioto (Geomagnetic Data Service). URL: http://wdc.kugi.kyoto-u.ac.jp/wdc/Sec3.html_(accessed 21.05.2017)

16. Caltech, Advanced Composition Explorer Science Center. URL: http://www.srl.caltech.edu/ACE/ASC/ (accessed 21.05.2017)

17. GOES (Geostationary Operational Environmental Satellite) Project site. URL: http://rsd.gsfc.nasa.gov/goes/ (accessed 21.05.2017) 